\title{
Exploring the Impact of Network Impairments on Remote Collaborative Augmented Reality Applications
}

\section{KEYWORDS}

Augmented Reality; Collaboration;

Network Impairments

\author{
Tooba Ahsen \\ Fahad R. Dogar \\ Tufts University \\ tooba.ahsen@tufts.edu \\ fahad@cs.tufts.edu
}

\author{
Aaron L. Gardony \\ CCDC - Soldier Center and CABCS \\ agardony@centerforabcs.org
}

\section{ABSTRACT}

Our research explores the impact of network impairments on remote augmented reality (AR) collaborative tasks, and possible strategies to improve user experience in these scenarios. Using a simple AR task, under a controlled network environment, our preliminary user study highlights the impact of network outages on user workload and experience, and how user roles and learning styles play a role in this regard.

\section{INTRODUCTION}

Given the importance of collaboration in our day-to-day lives, we are interested in augmented reality (AR) applications that can facilitate collaboration in a networked setting, when users may be located far away. Specifically, distance learning applications of various types (e.g., education, health, etc)

Permission to make digital or hard copies of part or all of this work for personal or classroom use is granted without fee provided that copies are not made or distributed for profit or commercial advantage and that copies bear this notice and the full citation on the first page. Copyrights for third-party components of this work must be honored. For all other uses, contact the owner/author(s)

CHI'19 Extended Abstracts, May 4-9, 2019, Glasgow, Scotland Uk

(c) 2019 Copyright held by the owner/author(s).

ACM ISBN 978-1-4503-5971-9/19/05...\$15.00

https://doi.org/10.1145/3290607.3312774 


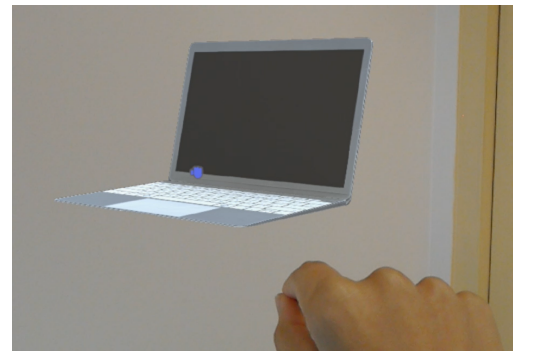

Figure 1: The user making a specific hand gesture to grab onto a virtual $3 \mathrm{D}$ object, and move it around.

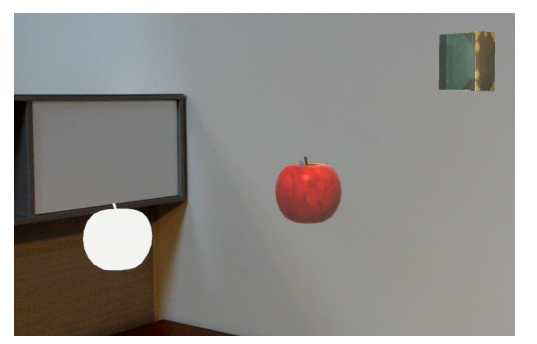

Figure 2: The guide's view through the headset. They see the starting/current positions of the objects (colored) and the target positions (white) but cannot move the objects.

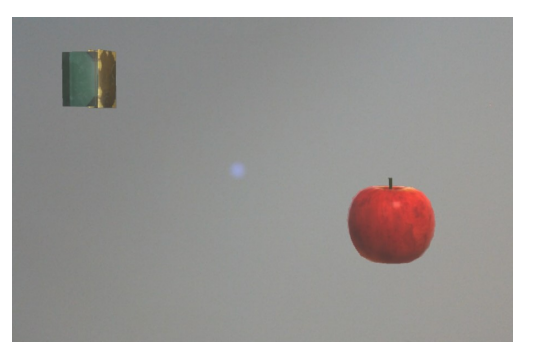

Figure 3: The manipulator's view through the headset. They see only the starting/current positions of the objects, and must follow the guide's instructions to move the objects to the target positions. could potentially provide a transformative experience to users. For example, in an at-home/distance physical therapy application, a patient could practise arm movement by using hand gestures to move, rotate, and scale, shared 3D objects, according to the instructions from the therapist who may be located far-away from the patient. Similarly, a group of students could effectively work together on a design project without requiring to be all in the same place.

To provide seamless exchange of audio and visual communication in the above scenarios, the role of the network (i.e., the Internet) becomes crucial. We posit that given the best-effort nature of today's Internet, network impairments are inevitable, and can play a crucial role in determining the effectiveness of AR-based remote collaboration. Network impairments include packet loss, high jitter, or outages, which could last from milliseconds to several seconds or minutes. Despite decades of research on network quality of service (QoS) $[4,12]$, anecdotal evidence as well as network measurement studies show that these impairments are still common [3, 8, 9].

The goal of our research is to understand how these network impairments impact AR-based collaborative experience and what could be possible strategies to improve user experience in these settings. To this end, we implement an AR-based collaborative application, and conduct a preliminary user study that provides insights on the impact of network impairments on user frustration and workload. Our insights show that user experience depends on the role of the users (e.g., manipulator vs. guide) in the collaborative task as well as the instruction style of different users. Based on these insights, we lay out a research agenda around substantiating our findings under a wider range of network impairments, and potential strategies to improve user experiences under network impairments, such as providing explicit feedback to the users about the duration of the outage.

\section{RELATED WORK}

Remote collaboration is a key usage scenario for AR: several studies have provided insights on different aspects of collaboration, such as increasing situational awareness using the gaze information of one's partner [2,13], the effectiveness of various pointing techniques in remote collaborative settings [1] or the importance of a shared view of the workspace in conversational grounding [5]. All these works introduce strategies to compensate for the lack of a shared real world environment (as in colocated tasks), so that collaboration does not suffer. However, little to no prior effort has looked into the impact of network impairments on user experience and effective collaboration in these settings.

In contrast, the networking/systems community has focused on more traditional interactive applications, such as video or voice conferencing $[8,11]$. There has been some work in exploring the impact of network impairments in single-user AR tasks, but not within a remote collaborative setting [15] In addition to audio and video communications, AR applications also involve users interacting with virtual content, within their real world environment, and users may encounter challenges related to establishing spatial orientations of their partners, different real world environments between users, 


\section{Table 1: Research Questions}

\begin{tabular}{|c|c|}
\hline RQ 1 & $\begin{array}{c}\text { What is the impact of network } \\
\text { impairments on user experience } \\
\text { in remote AR collaboration? }\end{array}$ \\
\hline RQ 2 & $\begin{array}{c}\text { Do user roles impact the severity with } \\
\text { which impairments are felt? }\end{array}$ \\
\hline
\end{tabular}

\section{Table 2: Guide's Workload (Nasa TLX} overall scores)

\begin{tabular}{|c|c|c|}
\hline Trial Type & Mean & Standard Deviation \\
\hline Without Outages & 35.037 & 23.291 \\
\hline With Outages & 53.556 & 22.232 \\
\hline
\end{tabular}

\section{Table 3: Manipulator's Workload} (Nasa TLX overall scores)

\begin{tabular}{|c|c|c|}
\hline Trial Type & Mean & Standard Deviation \\
\hline Without Outages & 38.185 & 19.226 \\
\hline With Outages & 43.852 & 18.986 \\
\hline
\end{tabular}

\section{Table 4: Guide's Frustration (Nasa TLX} frustration scale)

\begin{tabular}{|c|c|c|}
\hline Trial Type & Mean & Standard Deviation \\
\hline Without Outages & 27.778 & 24.509 \\
\hline With Outages & 59.444 & 28.988 \\
\hline
\end{tabular}

Table 5: Manipulator's Frustration (Nasa TLX frustration scale)

\begin{tabular}{|c|c|c|}
\hline Trial Type & Mean & Standard Deviation \\
\hline Without Outages & 21.111 & 19.965 \\
\hline With Outages & 45.000 & 30.311 \\
\hline
\end{tabular}

users getting disconcerted due to rapid 3D model manipulations, etc. While the former has been somewhat explored in the context of mixed space collaboration [6], the role of network impairments needs be further explored.

\section{PRELIMINARY USER STUDY}

We have designed an AR application (using Unity and the Mixed Reality Toolkit) that focuses on a collaborative problem solving task, and deployed it on the Microsoft HoloLens Head Mounted Display (HMD). The task involves two users (one guide and one manipulator) working together to solve a virtual 3D object positioning task, where objects have to be moved by the manipulator from their starting positions to target locations (using specific hand gestures - refer to Figure 1), based on the guide's instructions. The key point is that the target locations are only known to the guide, who needs to provide suitable instructions for the correct placement of the objects, while the objects can only be moved by the manipulator. Figure 2 and 3 show the different views of the guide and manipulator, respectively. Only through collaboration can they solve this task!

We emulate a remote setting by placing each user in separate rooms. The users are able to verbally communicate through their HMD and any object movement that the manipulator performs in their objects is seen by the guide. We emulate outages (longer periods of time with no network connectivity), which can last up to several seconds or minutes, as per network measurement studies [7] An outage results in disrupting both audio and visual communication, as no updates can be shared between users. Users perform the same object positioning task, with and without outages. Under an outage scenario, a total of 3 outages occur, in each of the 3 sub-tasks (every 3D object that the pair has to place in its target location is called a sub-task), where each outage lasts for 10 seconds each.

We chose a within-subject design for the study, with each pair of participants performing the task in both conditions. After each trial the participants were asked to fill in the NASA TLX questionnaire [10], in order to get a measure of their workload and frustration levels. At the end of the study, users filled out a questionnaire, and underwent a debriefing process. A total of 22 participants (11 pairs), of which two were pilot trials, participated in the IRB-approved (Institutional Review Board) study.

\section{HYPOTHESES}

Keeping in mind our higher level questions (see Table 1) and the observations made through pilot studies, we formulated the following hypotheses:

- Users experience an increase in their overall workload and frustration when performing a task in which network impairments occur, compared to the same task performed in perfect network conditions (in keeping with RQ 1).

- Outages have a greater impact on guides as compared to manipulators (in keeping with RQ 2). 
Table 6: Questionnaire responses highlighting the reasons for increased frustration during outages

\begin{tabular}{|c|c|}
\hline User & Response \\
\hline Manipulator (Pair 6) & $\begin{array}{c}\text { As a manipulator } \\
\text { I felt at a loss for what to } \\
\text { do and unsure what to do } \\
\text { to communicate to the guide } \\
\text { that I could no longer } \\
\text { respond to them. }\end{array}$ \\
\hline Manipulator (Pair 11) & $\begin{array}{c}\text { There were times } \\
\text { when I was in the middle of } \\
\text { moving an object in one } \\
\text { direction and I wouldn't be } \\
\text { sure if I should keep moving it } \\
\text { or not...I felt like we } \\
\text { had to keep asking each } \\
\text { other if we could hear } \\
\text { one another. }\end{array}$ \\
\hline Guide (Pair 4) & $\begin{array}{c}\text { Each break meant I had to } \\
\text { keep the instruction I was } \\
\text { about to say in mind, and } \\
\text { often had to say it several } \\
\text { times (first time not realizing } \\
\text { the other party could not hear) } \\
\text { and then again after the break } \\
\text { was over. }\end{array}$ \\
\hline
\end{tabular}

\section{RESULTS}

A one-tailed Paired T-test, with a confidence interval of $95 \%$, was used to evaluate the workloads. When individually evaluating the workload for guides and manipulators for scenarios with and without network impairments, we notice an increase in the workload of all the guides. This increase, we posit, stems from the fact that network impairments not only prevent guides from verbally communicating with their partners, but also prevent them from seeing how their partners manipulate objects, thus hindering their primary task all together. As can be seen in Table 2, there is a significant increase in the the mean value for guide's workload from the trial without outages, to the trial with outages $(t(8)=-4.402, p=0.001)$. One possible reason for this may be the increased frustration, experienced by guides due to the disruptions in communication caused by outages (see Table 4).

While some manipulators show a change in their workload under conditions of network impairment, this change varies across all manipulators. We attribute this variance to other features that influence the manipulator's workload, such as the physical demand involved in moving the objects using hand gestures. That said, looking at the frustration component, we see a significant increase in user frustration for tasks with outages than those without $(\mathrm{t}(8)=-2.676, \mathrm{p}=0.014)$ (see Table 5).

The above observations are also supported by the responses of the qualitative questionnaire. Users were asked to rate how disruptive outages were for their communication (from "Not Disruptive", to "Extremely Disruptive") using a 5 point Likert scale. Generally, guides tend to rate the disruptions higher (median $=4)$, as compared to manipulators (median $=3$ ). A Mann-Whitney $U$ test is used to establish that the difference in ratings is indeed significant $(U(8)=61, p=0.038)$.

Hence, the key takeaway is that network impairments lead to increased workload for the guides, possibly due to increased frustration. While manipulators experience increased frustration, no claims can be made regarding how their workload changes, as it is influenced by more than just frustration.

\section{DISCUSSION}

An analysis of the video recordings of the interaction between user pairs provides key insights into how roles, and learning/instruction styles impact the severity with which users experience outages.

\section{User Roles}

Within the video analysis, we noticed that manipulators often do not realize that outages have begun (if no verbal communication takes place), as they are engrossed in performing the manipulation Only when they ask questions or for further instructions, and receive no reply, do they realize that a network disruption may be underway. Guides on the other hand, are closely following the movements of objects, and often times follow the abrupt stop of the object's movements with inquiries or further instructions. When these go unanswered, guides are clued into the presence of outages. 


\section{Sidebar 1: Comments made by users about coping methods}

A: "Because I knew that they would happen I started to attend to relative and, where possible, absolute referents as a guide-post to enable the manipulator to do things autonomously. I found that absolute referents, such as doors, walls, and windows, were considerably more reliable than relative referents, such as distance." (guide, pair 7)

B: "It (outages) forced me to anticipate breaks in communication and communicate a batch of steps quickly to get across a complete picture so that despite a break in communication the manipulator might be able to continue in the right direction.” (guide, pair 3)

C: "We had to pause during the breaks. I went much slower with my movements to ensure that I was not messing up." (manipulator, pair 5)

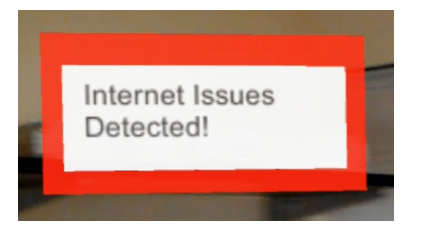

Figure 4: An example of a simple visual interface that can be used to explicitly inform users about the presence of an outage.

\section{Interaction Styles}

Based on video analysis, we see that the ways in which users interact with each other, within a collaborative setting, also varies. Some guides prefer to give general instructions, while others may give short step-by-step instructions to manipulators. Similarly, manipulators may check in with the guides, through verbal communication, to ensure that their actions are correct, or they may continue with the task and assume that the guide shall correct them, should the need arise. Shifting between instruction/learning styles (such as batching instructions) was one way users tried to cope with outages that occurred within the second task.

\section{How users naturally coped with outages}

Methods users adopted to cope with outages included using alternate spatial references, batching instructions and slowing down model movement.

Guides would sometimes react to outages by changing the spatial reference they used, so that manipulators were given enough information to autonomously move objects even when no communication was possible (Sidebar: 1A). Of course using referents like doors, walls etc, as mentioned in the quote, assumes that the users are aware of the terrain of their partner's surroundings, as well as their partner's orientation relative to objects in their surroundings. While this could be established over verbal communication, an easier strategy would be to use shared 3D objects (that both users can see) as spatial checkpoints, so that both users have a common understanding of distance [14].

Another strategy, adopted by the guides, was to batch instructions and relay them to the manipulators when they were sure that connection was restored (Sidebar: 1B). Meanwhile manipulators either continued following the last instruction they heard, or slowed their movements (Sidebar: 1C).

\section{RESEARCH AGENDA}

Our preliminary study focuses on understanding the impact of network impairments on collaborative AR tasks. Our ongoing work takes this work forward in two important ways.

First, we are evaluating the impact of different types of network impairments and their duration. Specifically, our preliminary study only focuses on a ten second outage - how do the results change if we have outages of smaller or longer duration.

Second, we are exploring strategies to mitigate the impact of outages on user experience and workload. This could involve giving users explicit information about the presence of outages, so that they can consciously adapt their collaborative behaviour, or using alternate communication mechanisms, such as using gaze information to convey focus/attention, instead of verbal communication during network impairments. Using role-specific interfaces has been somewhat explored in the context of Virtual Reality, and may be an effective strategy for Augmented Reality as well [16]. Moreover, if 


\section{ACKNOWLEDGEMENTS}

Research was partially sponsored by NSF (Award: 1618321) and the Combat Capabilities Development Command Soldier Center (under Cooperative Agreement Number W911QY-152-0001). The views and conclusions contained in this document are those of the authors and should not be interpreted as representing the official policies, either expressed or implied, of NSF, the Combat Capabilities Development Command Soldier Center, or the U.S. Government. The U.S. Government is authorized to reproduce and distribute reprints for Government purposes notwithstanding any copyright notation hereon. we can identify what instruction/learning styles work the best for situations with outages, it may be possible to use the timing and length of outages to drive users to change their interaction style into a more effective one. While we have performed some user studies where users are given information about outages using audio and visual interfaces (see Figure 4), we aim to corroborate our findings with an in-depth analysis of existing data, and additional user studies.

\section{REFERENCES}

[1] Deepak Akkil and Poika Isokoski. 2016. Accuracy of interpreting pointing gestures in egocentric view. In Proc. UbiComp '16. ACM.

[2] Deepak Akkil and Poika Isokoski. 2016. Gaze Augmentation in Egocentric Video Improves Awareness of Intention. In Proc. CHI'16. ACM.

[3] Alberto Dainotti, Claudio Squarcella, Emile Aben, Kimberly C Claffy, Marco Chiesa, Michele Russo, and Antonio Pescapé 2011. Analysis of country-wide internet outages caused by censorship. In Proc. IMC'11. ACM.

[4] Fahad R. Dogar, Laeeq Aslam, Zartash A. Uzmi, Sarmad Abbasi, and Young-Chon Kim. 2006. Connection Preemption in Multi-Class Networks. In IEEE GLOBECOM.

[5] Darren Gergle, Robert E Kraut, and Susan R Fussell. 2013. Using visual information for grounding and awareness in collaborative tasks. Human-Computer Interaction 28, 1 (2013), 1-39.

[6] Raphaël Grasset, Philip Lamb, and Mark Billinghurst. 2005. Evaluation of mixed-space collaboration. In Proc. ISMAR'05. IEEE Computer Society.

[7] Sarthak Grover, Mi Seon Park, Srikanth Sundaresan, Sam Burnett, Hyojoon Kim, Bharath Ravi, and Nick Feamster. 2013. Peeking Behind the NAT: An Empirical Study of Home Networks. In Proc. IMC '13.

[8] Osama Haq and Fahad R Dogar. 2015. Leveraging the power of cloud for reliable wide area communication. In Proc. ACM HotNets.

[9] Osama Haq, Mamoon Raja, and Fahad R Dogar. 2017. Measuring and improving the reliability of wide-area cloud paths. In Proc. WWW.

[10] Sandra G Hart and Lowell E Staveland. 1988. Development of NASA-TLX (Task Load Index): Results of empirical and theoretical research. In Advances in psychology. Vol. 52. Elsevier, 139-183.

[11] Junchen Jiang, Rajdeep Das, Ganesh Ananthanarayanan, Philip A Chou, Venkata Padmanabhan, Vyas Sekar, Esbjorn Dominique, Marcin Goliszewski, Dalibor Kukoleca, Renat Vafin, et al. 2016. Via: Improving internet telephony call quality using predictive relay selection. In Proc. SIGCOMM'16. ACM.

[12] Aadil Zia Khan, Shahab Baqai, and Fahad R Dogar. 2012. QoS aware path selection in content centric networks. In IEEE ICC. 2645-2649.

[13] Stephan Lukosch, Heide Lukosch, Dragos Datcu, and Marina Cidota. 2015. On the spot information in augmented reality for teams in the security domain. In Proc. CHI EA '15. ACM.

[14] Jens Müller, Roman Rädle, and Harald Reiterer. 2016. Virtual Objects as Spatial Cues in Collaborative Mixed Reality Environments: How They Shape Communication Behavior and User Task Load. In Proc. CHI'16. ACM.

[15] Mahdi Nabiyouni, Siroberto Scerbo, Doug A Bowman, and Tobias Höllerer. 2017. Relative effects of real-world and Virtual-World latency on an augmented reality Training Task: an ar simulation experiment. Frontiers in ICT 3 (2017), 34

[16] Lauriane Pouliquen-Lardy, Isabelle Milleville-Pennel, Francois Guillaume, and Franck Mars. 2016. Remote collaboration in virtual reality: asymmetrical effects of task distribution on spatial processing and mental workload. Virtual Reality 20 , 4 (2016), 213-220. 